\title{
Sliding Columnar Phase of DNA-Lipid Complexes
}

\author{
C.S. O'Hern and T.C. Lubensky \\ Department of Physics and Astronomy, University of Pennsylvania, Philadelphia PA 19104
}

\begin{abstract}
We introduce a simple model for DNA-cationic-lipid complexes in which galleries between planar bilayer lipid lamellae contain DNA 2D smectic lattices that couple orientationally and positionally to lattices in neighboring galleries. We identify a new equilibrium phase in which there are longrange orientational but not positional correlations between DNA lattices. We discuss properties of this new phase such as its x-ray structure factor $S(\mathbf{r})$, which exhibits unusual $\exp \left(-\operatorname{const.} \ln ^{2}|\mathbf{r}|\right)$ behavior as a function of in-plane separation $\mathbf{r}$.
\end{abstract}

DNA is a remarkable polymer that exhibits a complex phase behavior as a function of packing density, salt concentration, and other variables [1] . It is anionic, giving up positive counter ions to solution. Mixtures of DNA and cationic and neutral lipids in water form complexes that facilitate transfection of DNA into living cells and that play an important role in the emerging field of gene therapy [2]. Recent x-ray experiments [3] reveal the structure of these complexes at length scales from 10 to several hundred Angstroms, particularly near the isoelectric point where the total charge of counter ions given up by the DNA equals that given up by the cationic lipids. The lipids form bilayer membranes that stack in a lamellar structure (Fig. 1). Parallel strands of DNA arrange in 2D smectic structures in the galleries between lipid bilayers. The distance between lipid bilayers is equal to the diameter of a DNA molecule plus a hydration layer. In addition, the distance $d$ between DNA strands increases with increasing concentration of neutral lipids in a manner consistent with counter ions being expelled to solution and charge neutrality of the complex being determined only by the DNA and cationic lipids. The best fit to $\mathrm{x}$-ray diffraction data is obtained when some correlation between DNA lattices in different galleries is introduced.

We undertake here a theoretical investigation of possible equilibrium phases of these lamellar DNA-lipid complexes. We identify a new phase, with a nonvanishing smectic compression modulus $B$ in each gallery, in which there is long-range orientational but not positional correlation between DNA lattices in different galleries. This phase exhibits no restoring force for sliding DNA lattices rigidly relative to each other, but it does exhibit a restoring force preventing their relative rotation. We will refer to it as a sliding columnar phase. It is distinct from both the columnar phase in which the DNA segments form a 2D lattice and the totally decoupled phase in which there is no communication between different DNA lattices. It is similar to the decoupled phase of stacks of tethered membranes [ [ Dislocations can destroy positional correlations within the DNA lattices, melt the sliding columnar phase, and produce a nematic lamellar phase with $B=0$. Whether they always melt the phase will be discussed in detail elsewhere [5].

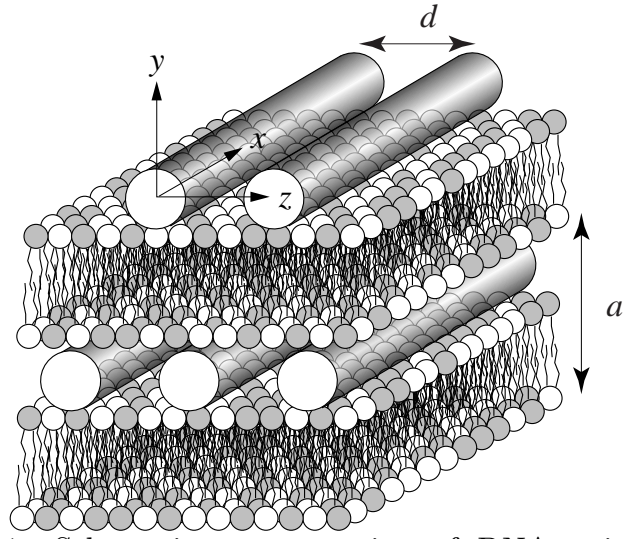

FIG. 1. Schematic representation of DNA-cationic-lipid complex. Parallel strands of DNA form smectic lattices with lattice spacing $d$ in galleries between lipid bilayers with spacing $a$. Charged and neutral lipid heads are, respectively, shaded and unshaded. DNA strands are aligned parallel to the $x$ axis, and the $y$ axis is the normal to the lipid planes.

We consider a model in which the DNA strands are confined to galleries between lipid bilayers in a perfect lamellar structure (with layer spacing $a$ ) with no dislocations or other defects. We assume the ground state of DNA strands in each gallery $n$ is that favored by electrostatic interactions, i.e., a 2D smectic lattice with layer spacing $d=2 \pi / k_{0}$. We take the lipid bilayers to be parallel to the $x z$ plane and the DNA strands to be aligned, on average, parallel to the $x$ axis as shown in Fig. 1. For the moment, we assume that the lipid bilayers are perfectly flat and do not fluctuate. In this case, long-wavelength properties of the DNA lattice in gallery $n$ are described entirely in terms of displacements $u^{n}(\mathbf{r})$ along the $z$ direction, where $\mathbf{r}=(x, z)$ is a position in the $x z$ plane. The Landau-Ginzburg-Wilson Hamiltonian for the complex is then a sum of independent elastic energies for each gallery and terms coupling displacements and angles in neighboring galleries: $\mathcal{H}=\mathcal{H}^{\mathrm{el}}+\sum_{n}\left(\mathcal{H}_{n}^{u}+\mathcal{H}_{n}^{\theta}\right)$ with

$$
\begin{aligned}
& \mathcal{H}^{\mathrm{el}}=\frac{1}{2} \sum_{n} \int d^{2} r\left[B_{2}\left(u_{z z}^{n}\right)^{2}+K_{2}\left(\partial_{x}^{2} u^{n}\right)^{2}\right] \\
& \mathcal{H}_{n}^{\theta}=-V_{\theta} \int d^{2} r \cos \left[2\left(\theta^{n}-\theta^{n+1}\right)\right]
\end{aligned}
$$




$$
\mathcal{H}_{n}^{u}=-V_{u} \int d^{2} r \cos \left[k_{0}\left(u^{n}-u^{n+1}\right)\right],
$$

where $\theta^{n} \approx \partial_{x} u^{n}$, and $u_{z z}^{n}=\partial_{z} u^{n}-\left[\left(\partial_{x} u^{n}\right)^{2}+\left(\partial_{z} u^{n}\right)^{2}\right] / 2$ is the nonlinear strain for gallery $n . B_{2}$ and $K_{2}$ are, respectively, the $2 D$ compression and bending moduli. $V_{u}$ is of order $\left(\lambda_{c}^{2} / d\right) e^{-2 \pi a / d}$ where $\lambda_{c}$ is the charge per unit length ( $e$ per $1.7 \AA$ ) of DNA. We do not yet have an estimate of $V_{\theta}$ whose dominant origin is likely a membrane mediated interaction.

If $V_{u}$ is sufficiently strong, the DNA strands form a regular $2 \mathrm{D}$ lattice in the $y z$ plane, and the whole complex will resemble an Abrikosov flux lattice in a high- $T_{c}$ material with the magnetic field parallel to the copper-oxide planes [6]. It will have the symmetry of an anisotropic discotic columnar liquid crystal [7]. As $V_{u}$ is reduced (or temperature is increased), fluctuations can melt the columnar DNA lattice without destroying the lipid-bilayer lamellar lattice. What is the nature of the melted phase? To answer this question, we consider the limit in which the potentials $V_{u}$ and $V_{\theta}$ are small. From the known statistical properties of $2 D$ smectics, we find that $V_{u}$ is irrelevant and $V_{\theta}$ is relevant with respect to the totally decoupled phase.

When the potentials $V_{\theta}$ and $V_{u}$ are zero, we have a stack of decoupled $2 D$ smectics, whose properties are by now well known [8,9. First we will ignore dislocations. At length scales less than the nonlinear lengths $l_{x}=K_{2}^{3 / 2} / T \sqrt{B_{2}}$ and $l_{z}=l_{x}^{2} / \lambda$, where $T$ is the temperature and $\lambda=\sqrt{K_{2} / B_{2}}$, fluctuations are described by the linearized elastic Hamiltonian with the nonlinear strain replaced by the linear strain $\partial_{z} u$. At lengths scales longer than $l_{x}$ and $l_{z}$, nonlinearities lead to renormalized bending and compression moduli $K(\mathbf{q})$ and $B(\mathbf{q})$ that, respectively, diverge and vanish at small wavenumber q. In both the harmonic and nonlinear regimes, the displacement correlation function in each gallery can be expressed as

$$
G(\mathbf{q})=q_{x}^{-\eta} Q\left(q_{z} / q_{x}^{\mu}\right) \sim \begin{cases}q_{x}^{-\eta}, & q_{z}=0 \\ q_{z}^{-\eta / \mu} & q_{x}=0 .\end{cases}
$$

In the harmonic regime, $\eta=4, \mu=2$, and $T G^{-1}(\mathbf{q})=$ $B_{2}\left(q_{z}^{2}+\lambda^{2} q_{x}^{4}\right)$. In the anharmonic regime, $\eta=7 / 2$, and $\mu=3 / 2$. The mean-square fluctuation in the displacement diverges in both regimes with the lengths $L_{x}$ and $L_{z}$ of the sample in the $x z$-plane:

$$
\left\langle u^{2}\right\rangle=\int \frac{d^{2} q}{(2 \pi)^{2}} G(\mathbf{q})=L_{x}^{2 \alpha} f_{u}^{(1)}\left(L_{z} / L_{x}^{\mu}\right),
$$

where $2 \alpha=\eta-1-\mu=1$ in both regimes and $f_{u}^{(1)}(0) \sim$ $T / \sqrt{K_{2} B_{2}}$ and $f_{u}^{(1)}(w) \sim \lambda^{2} l_{z}^{-2 \alpha / \mu} w^{2 \alpha / \mu}$ as $w \rightarrow \infty$. Alternatively the displacement correlation function,

$$
g_{u}(\mathbf{r})=\left\langle\left[(u(\mathbf{r})-u(0)]^{2}\right\rangle=|x|^{2 \alpha} f_{u}^{(2)}\left(|z| /|x|^{\mu}\right),\right.
$$

diverges with separation $\mathbf{r}$. Angular fluctuations are non divergent : $\left\langle\theta^{2}\right\rangle=\int \frac{d^{2} q}{(2 \pi)^{2}} q_{x}^{2} G(\mathbf{q})=\Lambda_{x}^{2 \alpha} f_{\theta}\left(\Lambda_{z} / \Lambda_{x}^{\mu}\right)$, where $\Lambda_{x} \sim 1 / d \sim \Lambda_{z} \sim 1 / d$ are the wavenumber cutoffs in $q_{x}$ and $q_{z}$ and $f_{\theta}(0)=$ const. and $f_{\theta}(w) \sim w^{2 \alpha / \mu}$.

Dislocations define another length scale $\xi_{2 D}^{\mathrm{d}}=n_{\mathrm{d}}^{-1 / 2} \approx$ $\exp \left(E_{2 D}^{\mathrm{d}} / 2 T\right)$, where $n_{\mathrm{d}}$ is the density of dislocations and $E_{2 D}^{\mathrm{d}}$ is the energy of a dislocation, which is finite for smectics. At length scales less than $\xi_{2 D}^{\mathrm{d}}$, the system is described by the harmonic or the nonlinear elastic theory. At length scales longer than $\xi_{2 D}^{\mathrm{d}}$, dislocations melt the smectic lattice, leaving a $2 D$ nematic with power-law angular correlations [8]. In what follows, we concentrate on the case in which $\xi_{2 D}^{\mathrm{d}}$ is the longest length scale in the problem and in which there is a regime in which dislocations can be ignored.

The coupling energies $\mathcal{H}_{n}^{\theta}$ and $\mathcal{H}_{n}^{u}$ are irrelevant if they tend to zero at large $L_{x}$ and $L_{z}$ and relevant if they diverge with $L_{x}$ and $L_{z}$. If DNA lattices are totally decoupled, $\left\langle\mathcal{H}_{n}^{u}\right\rangle=-V_{u} L_{x} L_{z} \exp \left(-k_{0}^{2}\left\langle u^{2}(\mathbf{r})\right\rangle\right)$ tends exponentially to zero for both harmonic and anharmonic $2 D$ elasticities, and is irrelevant. On the other hand $\left\langle\theta^{2}(\mathbf{r})\right\rangle$ is finite for both linear and nonlinear elasticity, and the potential $\left\langle\mathcal{H}_{n}^{\theta}\right\rangle=-V_{\theta} L_{x} L_{z} e^{-4\left\langle\theta^{2}\right\rangle}$ diverges with $L_{x} L_{z}$ and is relevant. When dislocations are allowed, then $\left\langle\cos \theta_{n}\right\rangle$ dies off as a power law at large $L_{x}$ and $L_{z}$, and $\left\langle\mathcal{H}_{n}^{\theta}\right\rangle$ may be relevant or irrelevant. Thus, if we ignore dislocations, the angular coupling is relevant, and the decoupled Hamiltonian will flow to a new long-wavelength Hamiltonian with angular but not positional coupling between layers. In the continuum limit, this Hamiltonian is

$$
\mathcal{H}=\frac{1}{2} \int d^{3} x\left[B u_{z z}^{2}+K\left(\partial_{x}^{2} u\right)^{2}+K_{y}\left(\partial_{y} \partial_{x} u\right)^{2}\right],
$$

where $\mathbf{x}=(\mathbf{r}, y), u(\mathbf{x})=u^{y / a}(\mathbf{r}), B=B_{2} / a, K=K_{2} / a$, and the nonlinear strain is still that of a two-dimensional system. Note that there are no terms proportional to $\left(\partial_{y} u\right)^{2}$ or $\left(\partial_{y}^{2} u\right)^{2}$. The only term involving $y$ derivatives is the one $K_{y}\left(\partial_{y} \partial_{x} u\right)^{2}=K_{y}\left(\partial_{y} \theta\right)^{2}$ coupling angles in different layers. This Hamiltonian is invariant under transformations of the type $u(\mathbf{x}) \rightarrow u(\mathbf{x})+f(y)$ for any function $f(y)$ independent of $\mathbf{r}$, i.e., it is invariant under arbitrary rigid displacements of one layer relative to another. To lowest order in $V_{\theta}, K_{y}=V_{\theta} a\langle\cos 2 \theta\rangle_{0}^{2}=V_{\theta} a e^{-4\left\langle\theta^{2}\right\rangle}$.

The elastic constant $K_{y}$ introduces a new length $l_{y}=$ $a \sqrt{K / K_{y}}$. At length scales within a gallery less than $l_{y}$, DNA lattices behave like independent $2 D$ smectics. If $l_{y}<l_{x, z}$, there will be a crossover from $2 D$ harmonic to $3 D$ sliding lattice at length scales of order $l_{y}$. If $l_{y}>l_{x, z}$, there will be a crossover first to $2 D$ nonlinear behavior and then to $3 D$ sliding behavior.

Fluctuations in $u$ in the harmonic limit are now determined by $G(\mathbf{q})=T\left[B q_{z}^{2}+K q_{x}^{4}+K_{y} q_{x}^{2} q_{y}^{2}\right]^{-1}$. They diverge with system size $L$ :

$$
\left\langle u^{2}\right\rangle \sim C \ln ^{2} L \text { and }\left\langle\left(\Delta u_{p}\right)^{2}\right\rangle=A_{p} C \ln L,
$$

where $\Delta u_{p}=u^{n+p}(\mathbf{r})-u^{n}(\mathbf{r}), C=T / \sqrt{B K_{y}}$, and $A_{p}$ is a number. Thus, $\left\langle\mathcal{H}_{n}^{u}\right\rangle \approx-V_{u} L^{2-\eta_{c}}$, where 
$\eta_{c}=A_{1} k_{0}^{2} C / 2$ tends to zero at large $L$ for $\eta_{c}>2$, and $V_{u}$ is irrelevant for $T>T_{u}=\left(4 / A_{1} k_{0}^{2}\right) \sqrt{B K_{y}}$.

Since $\left\langle u^{2}\right\rangle$ diverges with $L$, the correlation function $g_{u}(\mathbf{x})=\left\langle[u(\mathbf{x})-u(0)]^{2}\right\rangle$ diverges with $\mathbf{x}$. In the limit $L \rightarrow \infty, g_{u}(\mathbf{r}, 0)$ is finite for all $\mathbf{r}$ and at large $|\mathbf{r}|$ is

$$
g_{u}(\mathbf{r}, 0) \sim C \ln ^{2}\left(\Lambda^{2} \lambda|\mathbf{r}|\right) .
$$

When $y$ is nonzero, $g_{u}(\mathbf{r}, y)$ diverges with $L$ for all $y$. The behavior of $g_{u}(\mathbf{x})$ has interesting consequences for the x-ray structure factor $S(\mathbf{q})=\int d^{3} x S(\mathbf{x}) e^{-i \mathbf{q} \cdot \mathbf{x}}$, where $S(\mathbf{x})=\left\langle e^{i k_{0}[u(\mathbf{x})-u(0)]}\right\rangle$. For $y=0$, the dominant contribution to $S(\mathbf{r}) \equiv S(\mathbf{r}, 0)=\exp \left[-k_{0}^{2} g_{u}(\mathbf{r}, 0) / 2\right]$ is

$$
S(\mathbf{r})= \begin{cases}e^{-C k_{0}^{2}\left(\ln ^{2} \Lambda^{2} \lambda|\mathbf{r}|\right) / 2}, & |\mathbf{r}|>l_{y} \\ e^{-k_{0}^{2}|x| f_{u}^{(2)}\left(|z| /|x|^{\mu}\right) / 2}, & |\mathbf{r}|<l_{y} .\end{cases}
$$

The contribution to $S(0, y)$ arising from $g_{u}(0, y)$ is zero in the infinite volume limit when $V_{u}$ is irrelevant. There are, however, short-range positional correlations between layers arising from the irrelevant variable $V_{u}$. To lowest order in a perturbation expansion in $V_{u} / T, S(0, y=$ $n a) \sim\left(V_{u} / 2 T\right)^{n} I^{n}$ where $I$ has contributions of the form $\int d^{2} r e^{-k_{0}^{2} g_{u}(\mathbf{r}, 0) / 2}$. Thus, there is exponential decay of $S(0, y) \sim e^{-|y| / \xi_{y}}$ with $\xi_{y}=a / \ln \left(2 T / V_{u} I\right)$. The correlation length $\xi_{y}$ is finite so long as $V_{u} I<2 T$. Since $g_{u}(\mathbf{r}, 0)$ grows more rapidly with $\mathbf{r}$ in the totally decoupled phase than in the sliding phase, $I$ and $\xi_{y}$ are smaller in the latter than in the former phase. There are further contributions to $S(\mathbf{r}, y)$ that become more important as $I$ grows larger. When $\xi_{y}$ diverges, there will be a transition to the true columnar crystal phase with a nonvanishing $2 D$ shear modulus.

The Hamiltonian of Eq. (5) has nonlinear parts arising from the nonlinear strain $u_{z z}$. These lead to logarithmic renormalizations of the coefficients $B, K$, and $K_{y}$ similar to those in smectic liquid crystals [10]. We find

$$
K_{y}(\mathbf{q}) \sim K^{1 / 2}(\mathbf{q}) \sim B^{-1 / 3}(\mathbf{q}) \sim[\ln (\Lambda / h(\mathbf{q}))]^{1 / 4},
$$

where $h(\mathbf{q})=\left[q_{z}^{2}+\lambda^{2} q_{x}^{4}+\lambda_{y}^{2} q_{x}^{2} q_{y}^{2}\right]^{1 / 2}$ with $\lambda^{2}=K / B$ and $\lambda_{y}^{2}=K_{y} / B$. Preservation of rotational invariance in the calculation of the above renormalized elastic constants requires considerable care. Details of this calculation will be presented in a separate publication [11].

We have thus far ignored dislocations. In twodimensions, dislocations cause the smectic lattice to melt at length scales larger that $\xi_{d}^{2}$. Dislocations will certainly suppress order in the sliding phase. To obtain quantitave estimates of their effect, we need to calculate the energy per unit length of a dislocation predicted by the elastic energy of the sliding phase. We would like to obtain not only the elastic energy of a dislocation but also some estimate of its core energy, which can arise either from destruction of the order parameter $\psi$, describing periodic order, over an order-parameter coherence length $\xi$ or from deviations of the local director (specifying in this case the normal to the DNA strands) from the direction of preferred alignment over a bend or twist penetration depth $\lambda_{B}$. In the smectic- $A$ phase, screw dislocations have no elastic energy; their energy is totally in the core and is dominated in type II systems, for which $\lambda_{B}>\xi$, by director misalignment [12]. The mass-density amplitude $\psi_{\text {DNA }}$ for our DNA lattices is strictly speaking undefined in the sliding phase in the regions between DNA galleries. Thus, we argue that any core energy arising from dislocation lines parallel to the layers (i.e., in the $x z$ plane) must come from director mismatch rather than from destruction of periodic order. To describe director mismatch, we consider a "gauge" version of the elastic energy of the sliding phase in which displacements and angles are coupled in a rotationally invariant way:

$$
\begin{aligned}
\mathcal{H}_{g}= & \frac{1}{2} \int d^{3} x\left[B\left(\partial_{z} u\right)^{2}+D\left(\partial_{x} u-\theta\right)^{2}\right. \\
& \left.+K_{x}\left(\partial_{x} \theta\right)^{2}+K_{y}\left(\partial_{y} \theta\right)^{2}+K_{z}\left(\partial_{z} \theta\right)^{2}\right] .
\end{aligned}
$$

This energy reduces to the sliding phase elastic energy [Eq. (5)] when $\theta$ is integrated out. It introduces twist and bend penetrations depths $\lambda_{y}=\sqrt{K_{y} / D}$ and $\lambda_{z}=$ $\sqrt{K_{z} / D}$ characterizing length scales over which orientations relax in response to layer distortions.

Dislocations are topological line defects. They can be characterized by a density $\mathbf{b}(\mathbf{x})$ that determines the singular part of the displacement variable $u(\mathbf{x}): \boldsymbol{\nabla} \times$ $\nabla u(\mathbf{x})=\mathbf{b}(\mathbf{x})$. Using standard techniques 13, we can calculate dislocation energies in terms of the Fourier transform $\mathbf{b}(\mathbf{q})$ of $\mathbf{b}(\mathbf{x})$ :

$$
E=\frac{1}{2} B \int \frac{d^{3} q}{(2 \pi)^{3}} \frac{K(\mathbf{q}) q^{2}\left|b_{y}(\mathbf{q})\right|^{2}}{B q_{z}^{2}+K(\mathbf{q}) q^{2}\left[q_{x}^{2}+(B / D) q_{z}^{2}\right]},
$$

where $K(\mathbf{q}) q^{2}=K_{x} q_{x}^{2}+K_{y} q_{y}^{2}+K_{z} q_{z}^{2}$. This energy depends only on the $y$ component of $\mathbf{b}$, i.e., on the component of $\mathbf{b}$ normal to the bilayer planes. When there is a single dislocation line parallel to the $y$ axis with $\mathbf{b}(\mathbf{x})=d \mathbf{e}_{y} \delta(x) \delta(z)$, the energy per unit length $\epsilon$ predicted by this equation is precisely $E_{2 D}^{\mathrm{d}} / a$ where $E_{2 D}^{\mathrm{d}}$ is the energy of a two-dimensional edge dislocation. Since Eq. (11) includes orientational mismatch energy and we expect no core energy from destruction of the massdensity order parameter, we conclude that the energy for dislocations with $\mathbf{b}$ in the $x z$ plane is in fact zero. Thus, it is possible to form dislocation loops with arbitrarily long segments in the $x z$ plane. (This situation is analogous to that of the "pancake" models of flux lattices in high- $T_{c}$ superconductors [14]). These loops are equivalent to independent $2 D$ dislocations connected by loop segments with $b_{y}=0$ passing between DNA galleries. Dislocations will melt the sliding columnar phase if these $2 D$ dislocations melt the smectic lattice in any given gallery. Smectic lattices in galleries above and below a given gallery act as external fields orienting that gallery along the common direction of the whole sample. Thus, each gallery is equivalent to a smectic in an external aligning field (whose long-wavelength Hamiltonian is 
the in the universality class of the $x y$ model [13]) that will Kosterlitz-Thouless melt to a nematic at a temperature $T_{N}$. If $T_{N}>T_{u}$, then for $T_{u}<T<T_{N}$, there lattices have not melted, $V_{u}$ is irrelevant, and the sliding columnar phase exists. If $T_{u}>T_{N}$, then the sliding columnar phase only exists up to length scales of order the KT correlation length $\xi_{N}=d \exp \left(b /\left|T-T_{N}\right|^{1 / 2}\right)$, where $b$ is a nonuniversal constant. Beyond $\xi_{N}$, the phase will be a lamellar nematic. The possibility of $T_{u}<T_{N}$ will be addressed elsewhere [5].

In our discussion to this point, we have assumed that lipid bilayers define absolutely rigid $2 D$ galleries for the DNA. In reality, the lipid bilayers fluctuate. To treat these fluctuations, we should introduce a height variable $h^{n}(\mathbf{r})$ for membrane $n$ and a $y$-displacement variable $u_{y}^{n}(\mathbf{r})$ for the DNA lattice in the $n$th gallery. Clearly the variables are coupled, and at long wavelengths, we can assume they are locked together. Thus, we can construct a continuum elastic hamiltonian in terms of $u(\mathbf{x}) \equiv u_{z}(\mathbf{x})$ and $u_{y}(\mathbf{x})$ :

$$
\begin{aligned}
\mathcal{H}_{y z}= & \frac{1}{2} \int d^{3} x\left[B^{z} u_{z z}^{2}+K_{x x}^{z}\left(\partial_{x}^{2} u_{z}\right)^{2}+K_{x y}^{z}\left(\partial_{x} \partial_{y} u_{z}\right)^{2}\right. \\
& +B^{y} u_{y y}^{2}+2 B^{y z} u_{z z} u_{y y} \\
& \left.+K_{x x}^{y}\left(\partial_{x}^{2} u_{y}\right)^{2}+2 K_{x z}^{y}\left(\partial_{x} \partial_{z} u_{y}\right)^{2}+K_{z z}^{y}\left(\partial_{z}^{2} u_{y}\right)^{2}\right] .
\end{aligned}
$$

The DNA lattice introduces a preferred direction in the $x z$ plane that causes the bend elastic constant tensor $K_{i j}^{y}$ for $u_{y}$ to be anisotropic. The statistical properties of $u_{z}$, including the correlation function $S(\mathbf{q})$, predicted by this Hamiltonian are essentially identical to those predicted by the simpler rigid-layer Hamiltonian of Eq. (11). The Grinstein-Pelcovits renormalization of $B^{z}, K_{x x}^{z}$ and $K_{x y}^{z}$ are identical to those of $B, K$, and $K_{y}$. We can use $\mathcal{H}_{y z}$ to calculate smectic correlations in the lipid bilayers determined by $S_{h}(\mathbf{x})=\left\langle e^{i q_{0}\left[u_{y}(\mathbf{x})-u_{y}(0)\right]}\right\rangle$, where $q_{0}=2 \pi / a$. X-ray diffraction measures $S_{h}(\mathbf{q})$, the Fourier transform of $S_{h}(\mathbf{x})$, as well as $S(\mathbf{q})$. The most noticeable difference between this function and that of standard lamellar phases arises from the anisotropy in the bending modulus. There are other differences arising from the $u_{z z}-u_{y y}$ coupling that will be discussed in more detail in an upcoming publication.

The intrinsic chirality of DNA molecules leads to interactions that will cause the direction of DNA lattices to rotate, like the director in a cholesteric phase, about an axis perpendicular to the layers in both the sliding columnar and lamellar nematic phases. We expect, however, these interactions to be small and resultant pitches to be very large because the lipid bilayer prevents close approach of DNA molecules.

In this letter, we have introduced and investigated some of the properties of a new phase of matter that may exist in highly anisotropic, rotationally invariant columnar systems such as DNA-cationic-lipid complexes. It would be interesting to find evidence for this phase in the DNA complexes studied in Ref. [3]. The material in these experiments consisted of micron-size spherulites composed of apparently randomly oriented domains of length of order $L=500 \AA$ on a side of DNA-lipid-complex. The $\mathrm{x}$-ray data are consistent with nearly independent harmonic smectic layers with some exponentially attenuated positional correlation between layers. A nonvanishing $V_{u}$ will lead to exponential correlations between layers even in the harmonic regime. $500 \AA$ corresponds approximately to the nonlinear $2 D$ length $l_{x}$. Thus, it is not clear whether the domain size is set by actual distortions of the lipid layers or by the breakdown of harmonic behavior at length scales longer than $l_{x}$ or $l_{z}$. It will be necessary to prepare monodomain samples to determine whether there is a crossover to anharmonic $2 D$ behavior or to that of the $3 D$ sliding phase proposed in this paper.

We are grateful to Cyrus Safinya for inspiring our interest in this problem, to John Toner for emphasizing the importance of dislocation interactions in the sliding phase, and to Robijn Bruinsma and Randy Kamien for helpful discussions. This work was supported in part by the NSF under grant No. DMR94-23114.

Note added Results essentially identical to those reported here were obtained independently by Golubović and Golubović as reported in Ref. 15].

[1] F. Livolant, Physica A 176, 117 (1991).

[2] P. A. Felgner et al., Proc. Natl. Acad. Sci, USA 84, 7413 (1987).

[3] J. O. Rädler, I. Koltover, T. Salditt, and C. R. Safinya, Science 275, 810 (1997); T. Salditt, I. Koltover, J. Rädler, and C. Safinya, Phys. Rev. Lett. 79, 2582 (1997).

[4] J. Toner, Phys. Rev. Lett. 64, 1741 (1990); L. Golubović and T.C. Lubensky, Phys. Rev. A 43, 6793 (1991).

[5] C.S. O'Hern, T.C. Lubensky, and J. Toner (unpublished)

[6] G. Blatter et al., Rev. Mod. Phys. 66, 1125 (1994).

[7] P. de Gennes and J. Prost, The Physics of Liquid Crystals (Clarencon Press, Oxford, 1993).

[8] J. Toner and D. Nelson, Phys. Rev. B 23, 316 (1981).

[9] L. Golobović and Z.-G. Wang, Phys. Rev. E 49, 2567 (1994).

[10] G. Grinstein and R. Pelcovits, Phys. Rev. Lett. 47, 856 (1981); Phys. Rev. A 26, 915 (1982).

[11] C.S. O'Hern and T.C. Lubensky (unpublished).

[12] T.C. Lubensky, A.R. Day, and A.J. McKane, Phys. Rev. A 27, 1461 (1983).

[13] P. Chaikin and T. Lubensky, Principles of Condensed Matter Physics (Cambridge University Press, Cambridge, 1995).

[14] M. Feigel'man, V. Geshkenbein, and A. Larkin, Physica C 167, 177 (1990); S. Sengupta et al., Phys. Rev. Lett. 67, (1991).

[15] L. Golubović and M. Golubović, Phys. Rev. Lett. 80, 4341 (1998). 